\title{
Urea nitrogen, creatinine, and uric acid levels in postmortem serum, vitreous humor, and pericardial fluid
}

\author{
Cristian Palmiere • Patrice Mangin
}

Received: 21 May 2014 / Accepted: 28 August 2014 / Published online: 7 September 2014

(C) Springer-Verlag Berlin Heidelberg 2014

\begin{abstract}
Urea nitrogen, creatinine, and uric acid are relatively stable in postmortem serum and may, therefore, be used for diagnostic purposes when chronic kidney disease and end-stage renal failure are investigated as causes of death. Nevertheless, uncertainties remain in defining the best alternative to postmortem serum for the identification and assessment of significantly decreased kidney function. In this study, we investigated urea nitrogen, creatinine, and uric acid levels in postmortem serum, pericardial fluid, and vitreous humor in a series of medico-legal cases (500 autopsies) with various causes of death. No postmortem interval-related differences were observed in any of the investigated fluids for any analyzed parameter, confirming the biochemical stability of all compounds after death. Data analysis failed to reveal statistically significant differences between postmortem serum and pericardial fluid urea nitrogen, creatinine, and uric acid concentrations. Conversely, statistically significant differences were observed in all analyzed biomarkers between postmortem serum and vitreous humor levels, with lower concentrations of all markers measured in vitreous. The results of this study suggest that, in order to estimate as accurately as possible blood analyte concentrations at the time of death, pericardial fluid should be preferred to vitreous humor.
\end{abstract}

Keywords Postmortem biochemistry $\cdot$ Urea nitrogen · Creatinine $\cdot$ Uric acid $\cdot$ Chronic kidney disease $\cdot$ Pericardial fluid

\section{Introduction}

Chronic kidney disease (CKD) is a general term for heterogeneous disorders affecting kidney structure and function $[1,2]$.

C. Palmiere $(\bowtie) \cdot$ P. Mangin

University Centre of Legal Medicine, Rue du Bugnon 21,

1011 Lausanne, Switzerland

e-mail: cristian.palmiere@chuv.ch
The glomerular filtration rate (GFR) is the best overall index of kidney function and would ideally be measured with reference procedures that follow the clearance of an infused exogenous substance. Urinary inulin clearance is the gold standard for measuring GFR, though it is not routinely performed. A number of different tracers, both radioactive and nonradioactive, as well as different protocols, are used as alternatives to classic urinary inulin clearance. Regardless of methodology, these techniques are expensive and cumbersome, thereby rending them seldom performed in the clinical setting. As a result, GFR is far more commonly assessed using a variety of different endogenous markers such as urea nitrogen and creatinine and by means of equations [1, 3-8].

It has been repeatedly shown that GRF evaluation using serum creatinine may lead to overestimation of the true GFR in stage $5 \mathrm{CKD}$ due to creatinine tubular secretion. To overcome this limitation, an average of urea and creatinine clearance has been proposed to better estimate GFR in advanced CKD. The rationale for this is that urea clearance underestimated GRF as the molecule is passively reabsorbed in the proximal tubule. This GRF underestimation on behalf of urea will balance out GRF overestimation based on creatinine clearance $[1,7,8]$.

Urea nitrogen, creatinine, and uric acid have been demonstrated to be relatively stable in postmortem serum collected during autopsy and, therefore, useful for diagnostic purposes [9-13]. Numerous investigations have focused on urea nitrogen, creatinine, and uric acid levels in alternative biological samples (vitreous humor, synovial, pericardial, and cerebrospinal fluids) collected during autopsy, with results often interesting [9-30].

Nevertheless, uncertainties remain in defining the best alternative to postmortem serum in forensic pathology routine for the identification and assessment of significantly decreased kidney function. Indeed, no study as of yet has conclusively demonstrated that urea nitrogen, creatinine, and uric 
acid concentrations in postmortem serum, vitreous humor, and pericardial fluid are reciprocally correlated.

The aim of this study was to measure urea nitrogen, creatinine, and uric acid levels in postmortem serum obtained from femoral blood in a series of cases that underwent medico-legal autopsies and compare the obtained values to pericardial fluid and vitreous urea nitrogen, creatinine, and uric acid concentrations. Our goal was to evaluate whether both vitreous humor and pericardial fluid provide suitable results comparable to those found in postmortem serum.

\section{Materials and methods}

\section{Study protocol}

The present study was a prospective, single centre study, performed at the University Centre of Legal Medicine in Lausanne, Switzerland, during 2008-2013. All cases collected for this study underwent medico-legal autopsies as requested by the inquiring authorities. Laboratory analyses were performed as part of the medico-legal investigations.

\section{Population and postmortem investigations}

Postmortem serum from femoral blood, pericardial fluid, and vitreous humor were systematically collected from consecutive deceased subjects after their arrival at the morgue (vitreous humor), immediately prior to autopsy (femoral blood) or during postmortem examination (pericardial fluid). In total, 500 cases with various causes of death were included in this study (350 males and 150 females, 16-88 years of age). Postmortem intervals ranged between $8 \mathrm{~h}$ and $48 \mathrm{~h}$. Samples from severely decomposed corpses were rejected. Samples from cases with cranial destruction or chest trauma were not collected. Case inclusion criteria consisted of a postmortem interval not exceeding 48 hours and the availability of postmortem serum from femoral blood, vitreous humor, and pericardial fluid during postmortem investigations. The causes of death included acute traumatic deaths (80), mechanical asphyxiation (20), hypothermia (10), anaphylaxis (5), drug intoxication (180), bacterial sepsis (20), natural diseases (sudden cardiac deaths without acute coronary thrombosis, severe coronary atherosclerosis without acute coronary thrombosis, acute coronary thrombosis with myocardial infarction, 170 cases), and CKD (15 cases). No cases of hyperthermia (heat stroke) or gastrointestinal bleeding were collected.

\section{Sample collection}

Femoral blood samples were collected by aspiration with a sterile needle and syringe from the femoral vein(s) immediately prior to autopsy. Blood samples were drawn after clamping the vein(s) at the proximal end and lifting the lower $\operatorname{limb}(\mathrm{s})$ for several minutes. Blood was stored in tubes containing a gel serum separator. Blood was centrifuged immediately post collection at $3,000 \mathrm{~g}$ for $15 \mathrm{~min}$. After centrifugation, the separated supernatant (postmortem serum, between $1 \mathrm{ml}$ and $5 \mathrm{ml}$ ) was collected and stored in preservative-free tubes. No specimens were excluded due to insufficient sample volume. All samples were transferred to the laboratories immediately post collection. When analyses were delayed, samples were stored at $-20^{\circ} \mathrm{C}$.

Undiluted samples of pericardial fluid (between $5 \mathrm{ml}$ and $10 \mathrm{ml}$ ) were collected immediately post pericardium incision during autopsy. All samples were immediately centrifuged at $3,000 \mathrm{~g}$ for $15 \mathrm{~min}$. After centrifugation, the separated supernatant was collected and stored in preservative-free tubes. No specimens were excluded due to insufficient sample volume. All samples were transferred to the laboratories immediately post collection. When analyses were delayed, samples were stored at $-20^{\circ} \mathrm{C}$.

Undiluted vitreous humor samples (between 1 and $3 \mathrm{ml}$ ) were obtained by aspiration using a sterile needle and syringe. Right and left vitreous samples were collected through a scleral puncture at the lateral canthus, aspirated from the centre of each eye, pooled in the same syringe and mixed together. After collection, vitreous samples were immediately centrifuged at 3,000 g for 15 minutes. The separated supernatant was collected and stored in preservative-free tubes. No specimens were excluded due to insufficient sample volume. All samples were transferred to the laboratories immediately post collection. When analyses were delayed, samples were stored at $-20^{\circ} \mathrm{C}$.

\section{Laboratory assays}

Creatinine (Jaffé method, rate-blanked and compensated), urea nitrogen (kinetic enzymatic UV assay for urea/urea nitrogen) and uric acid (enzymatic uricase colorimetric AU Plus) were determined using the Roche standard methods on the Roche Modular P system (Roche Diagnostics GmbH, Mannheim, Germany). The following clinical reference intervals were considered for the analyzed markers: urea nitrogen $2.9-7.7 \mathrm{mmol} / \mathrm{l}(8.1-21.6 \mathrm{mg} / \mathrm{dl})(\mathrm{male})$ and $2.9-6.4 \mathrm{mmol} / \mathrm{l}$ $(8.1-17.9 \mathrm{mg} / \mathrm{dl})$ (female); creatinine $62-106 \mu \mathrm{mol} / \mathrm{l}(0.70$ $1.20 \mathrm{mg} / \mathrm{dl})(\mathrm{male})$ and $44-80 \mu \mathrm{mol} / \mathrm{l}(0.50-0.90 \mathrm{mg} / \mathrm{dl})$ (female); uric acid 202-416 $\mu \mathrm{mol} / 1$ (3.4-7.0 mg/dl) (male), and $142-339 \mu \mathrm{mol} / \mathrm{l}(2.4-5.7 \mathrm{mg} / \mathrm{dl})$ (female).

\section{Statistical analysis}

Nonparametric tests were used throughout the study. The Mann-Whitney U test was used to evaluate concentration differences of the tested parameters in collected biological fluids. Statistical significance was set at $\mathrm{p}<0.05$ for all tests. 
Graphpad Prism 4.0 (Graphpad Software, La Jolla, CA, USA) was used for statistics.

\section{Results}

The graphical representation of parameter distributions in postmortem serum, vitreous humor and pericardial fluid (box plots) is reported in Fig. 1. Table 1 summarizes ranges, mean values, medians and standard deviations for all tested parameters in all analyzed fluids. The main statistical analysis results are reported in Table 2.

Statistically significant differences were noted between males and females in all tested parameters and analyzed samples, with higher levels in males. No postmortem interval-related differences were observed in any of the investigated specimens for any analyzed parameter, confirming the biochemical stability of all compounds after death.

Urea nitrogen, creatinine, and uric acid concentrations showed similar distributions in all tested samples in relation to the causes of death: CKD and sepsis cases with multiple organ dysfunction syndrome had significantly higher levels of all measured biomarkers in all analyzed biological fluids compared to other groups.

Data analysis failed to reveal statistically significant differences between postmortem serum and pericardial fluid urea nitrogen, creatinine, and uric acid concentrations. Conversely, statistically significant differences were observed in all analyzed biomarkers between postmortem serum and vitreous humor levels, with lower concentrations of all markers measured in vitreous. The less statistically significant difference pertained to urea nitrogen ( $\mathrm{p}$ value 0.0229 ).

\section{Discussion}

Urea nitrogen, creatinine and uric acid have been demonstrated to be relatively stable in postmortem serum collected during autopsy and, therefore, to be reliable for diagnostic purposes when kidney disease is investigated as the cause of death [9-13].

Analyses performed in cerebrospinal fluid revealed slight rises of urea nitrogen after death. However, the measured values appeared to reliably reflect antemortem levels as they apparently do in vitreous humor and synovial fluid. Analogously, creatinine concentrations proved stable in vitreous and cerebrospinal fluid after death [16, 18-25, 27, 29, 30].

Pericardial fluid urea nitrogen, creatinine, and urate can be collected within 48 hours postmortem, with levels unrelated to postmortem intervals [10, 14, 15, 17]. Conversely, significant changes seem to occur in electrolyte and enzyme values in relation to the postmortem period, which may represent a

\section{Urea nitrogen}

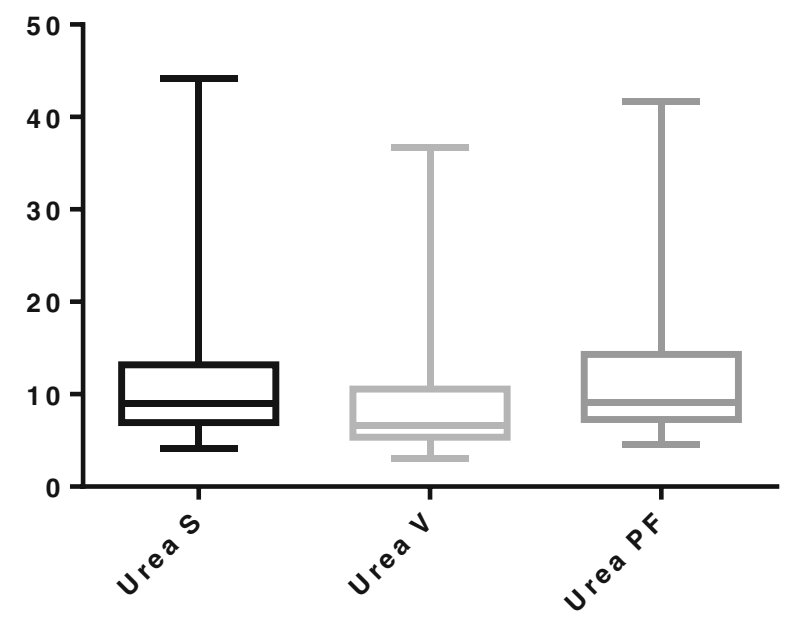

Creatinine

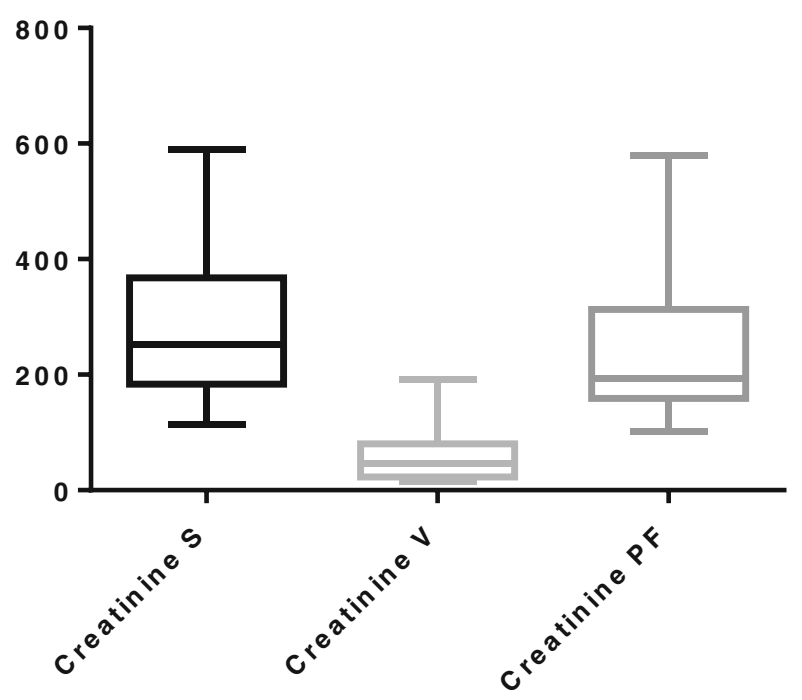

Uric acid

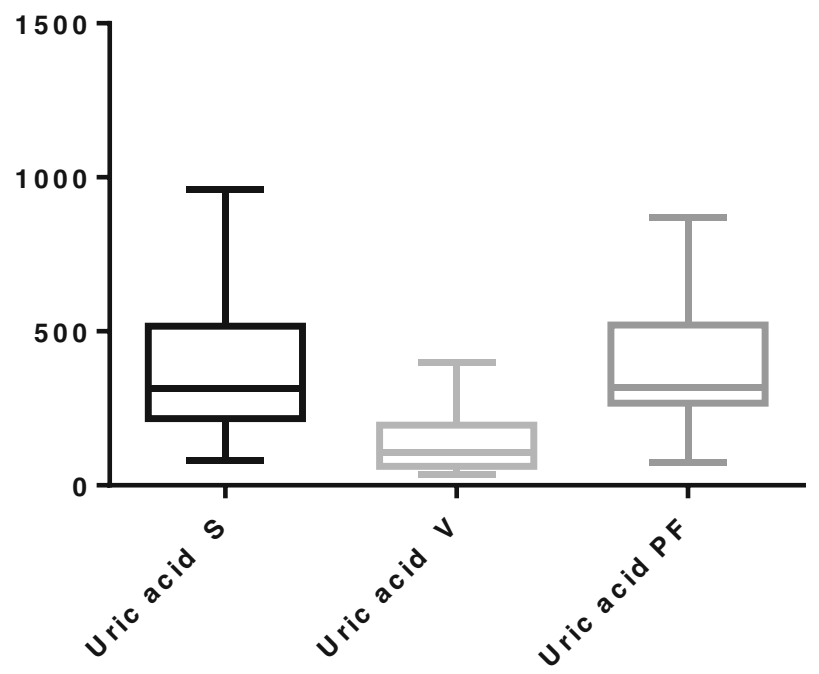

Fig. 1 Graphical representation (box plots) of parameter distributions in postmortem serum (S), vitreous humor (V) and pericardial fluid (PF) 
Table 1 Summarizes ranges, mean values, medians, and standard deviations for all tested parameters in all analyzed fluids. Results are expressed in $\mathrm{mmol} / \mathrm{l}$ (urea nitrogen) and $\mu \mathrm{mol} / \mathrm{l}$ (creatinine and uric acid)

\begin{tabular}{llll}
\hline & Urea nitrogen & Creatinine & Uric acid \\
\hline Blood reference value male & $2.9-7.7$ & $62-106$ & $202-416$ \\
Blood reference value female & $2.9-6.4$ & $44-80$ & $142-339$ \\
Postmortem serum & & & \\
$\quad$ Range & $4.2-44.2$ & $110-589$ & $82-959$ \\
Mean value & 13.2 & 264 & 213 \\
Median & 9.0 & 237 & 305 \\
Standard deviation & 10.6 & 116 & 178 \\
Vitreous humor & & & \\
Range & $3.0-36.7$ & $15-191$ & $30-400$ \\
Mean value & 10.6 & 59 & 138 \\
Median & 6.6 & 44 & 110 \\
Standard deviation & 9.2 & 47 & 105 \\
Pericardial fluid & & & \\
Range & $4.6-41.7$ & $90-579$ & $74-869$ \\
Mean value & 13.8 & 225 & 374 \\
Median & 9.1 & 196 & 317 \\
Standard deviation & 10.9 & 106 & 166 \\
\hline
\end{tabular}

limitation for some pericardial fluid analysis application in forensic pathology routine [31-41]. Moreover, urea nitrogen, creatinine, and urate concentrations in pericardial fluid are independent of the amount of the sampled volume, indicating no significant interference of postmortem water redistribution. Based on the results of former studies, it was postulated that pericardial fluid urea nitrogen, creatinine, and urate could be regarded as suitable biochemical markers for the pathophysiological investigation of situations in which dehydration, enhanced protein catabolism due to severe catabolic states, gastrointestinal bleeding, skeletal damage, and impaired renal function might be involved. Specific patterns of normal/ increased pericardial fluid urea nitrogen, creatinine, and urate would, therefore, suggest or reflect (or conversely rule out) systemic organ tissue damage involving skeletal muscles, complications of renal dysfunction, progressive systemic deterioration involving renal failure, and survival time-

Table 2 Summarizes the results of statistical analyses. For all tests, statistical significance was set at $\mathrm{p}<0.05$

\begin{tabular}{ll}
\hline & $\mathrm{p}$ value \\
\hline Urea nitrogen postmortem serum vs. vitreous humor & 0.0229 \\
Urea nitrogen postmortem serum vs. pericardial fluid & 0.5781 \\
Creatinine postmortem serum vs. vitreous humor & $<0.001$ \\
Creatinine postmortem serum vs. pericardial fluid & 0.1013 \\
Uric acid serum vs. vitreous humor & $<0.001$ \\
Uric acid postmortem serum vs. pericardial fluid & 0.4931 \\
\hline
\end{tabular}

dependent increases in biomarker concentrations indicating prolonged death processes. In this regard, comparisons between pericardial fluid and postmortem serum urea nitrogen, creatinine, and urate levels have been proposed as highly useful for investigating the cause and process of death to reinforce pathological and toxicological findings $[10,14,15$, 17].

The results of our investigations seem to indicate that pericardial fluid and postmortem serum creatinine, urea nitrogen, and uric acid levels significantly correspond, thus, allowing blood concentrations at the time of death to be estimated.

Blood clinical reference values for creatinine, urea nitrogen, and uric acid could, therefore, be applied to postmortem pericardial fluid.

Globally considered, these findings concur with previous observations on this topic and corroborate the usefulness of pericardial fluid analysis for urea nitrogen, creatinine, and uric acid determination. Pericardial fluid can, therefore, be regarded as a valid alternative to postmortem serum when femoral blood is unavailable at autopsy and CKD (or other clinical situations characterized by increased urea nitrogen, creatinine or uric acid) must be investigated.

On the other hand, statistically significant differences were observed in all analyzed biomarkers between postmortem serum and vitreous humor levels, with lower concentrations of all markers measured in vitreous. These findings would suggest that elevated vitreous humor values of these analytes can be of diagnostic significance. Nevertheless, in order to estimate as accurately as possible blood analyte concentrations at the time of death, pericardial fluid should be preferred to vitreous humor.

Based on the results of our investigations, vitreous creatinine and uric acid do not appear to adequately reflect corresponding levels in postmortem serum and, thus, antemortem blood levels. Concerning estimation of blood urea nitrogen concentration at the time of death, pericardial fluid urea nitrogen levels should be preferred to vitreous humor levels, which may be used though with caution.

To conclude, urea nitrogen, creatinine, and uric acid concentrations in vitreous humor do not seem to be as reliable as pericardial fluid levels in estimating antemortem blood concentrations. Vitreous humor should, therefore, be used for the postmortem determination of biomarkers that have been demonstrated to be minimally influenced by postmortem changes and whose vitreous concentrations seem to be, at present, the most comparable to presumed antemortem blood levels.

Role of funding source This study was not financially supported.

Conflict of interest The authors disclose any financial and personal relationship with other people or organizations that could potentially or inappropriately influence their work and conclusions. 


\section{References}

1. Levey AS, Coresh J (2012) Chronic kidney disease. Lancet 379:165-180

2. Wen CP, Cheng TY, Tsai MK, Chang YC, Chan HT, Tsai SP, Chiang PH, Hsu CC, Sung PK, Hsu YH, Wen SF (2008) All-cause mortality attributable to chronic kidney disease: a prospective cohort study based on 462293 adults in Taiwan. Lancet 371:2173-2182

3. Ortega LM, Arora S (2012) Metabolic acidosis and progression of chronic kidney disease: incidence, pathogenesis, and therapeutic options. Nefrologia 32:724-730

4. Eknoyan G (2007) Chronic kidney disease definition and classification: the quest for refinement. Kidney Int 72:1183-1185

5. Winearls CG, Haynes R, Glassock R (2010) CKD staging - evolution not revolution. Nefrologia 30:493-500

6. Turner JM, Bauer C, Abramowitz MK, Melamed ML, Hostetter TH (2012) Treatment of chronic kidney disease. Kidney Int 81:351-362

7. White CA, Akbari A (2011) The estimation, measurement, and relevance of the glomerular filtration rate in stage 5 chronic kidney disease. Semin Dial 24:540-549

8. Lamb EJ, Stevens PE (2014) Estimating and measuring glomerular filtration rate: methods of measurement and markers for estimation. Curr Opin Nephrol Hypertens 23:258-266

9. Zhu BL, Ishida K, Quan L, Taniguchi M, Oritani S, Li DR, Fujita MQ, Maeda H (2002) Postmortem serum uric acid and creatinine levels in relation to the causes of death. Forensic Sci Int 125:59-66

10. Zhu BL, Ishikawa T, Michiue T, Tanaka S, Zhao D, Li DR, Quan L, Oritani S, Maeda H (2007) Differences in postmortem urea nitrogen, creatinine and uric acid levels between blood and pericardial fluid in acute death. Legal Med (Tokyo) 9:115-122

11. Uemura K, Shintani-Ishida K, Saka K, Nakajima M, Ikegaya H, Kikuchi Y, Yoshida K (2008) Biochemical blood markers and sampling sites in forensic autopsy. J Forensic Legal Med 15:312-317

12. Coe JI (1993) Postmortem chemistry update. Emphasis on forensic application. Am J Forensic Med Pathol 14:91-117

13. Maeda H, Zhu BL, Bessho Y, Ishikawa T, Quan L, Michiue T, Zhao D, Li DR, Komatsu A (2008) Postmortem serum nitrogen compounds and C-reactive protein levels with special regard to investigation of fatal hyperthermia. Forensic Sci Med Pathol 4:175-180

14. Zhu BL, Ishikawa T, Michiue T, Li DR, Zhao D, Quan L, Maeda H (2005) Evaluation of postmortem urea nitrogen, creatinine and uric acid levels in pericardial fluid in forensic autopsy. Legal Med (Tokyo) 7:287-292

15. Kawamoto O, Michiue T, Ishikawa T, Maeda H (2013) Comprehensive evaluation of pericardial biochemical markers in death investigation. Forensic Sci Int 224:73-79

16. Balasooriya BA, St Hill CA, Williams AR (1984) The biochemistry of vitreous humour. A comparative study of the potassium, sodium and urate concentrations in the eyes at identical time intervals after death. Forensic Sci Int 26:85-91

17. Arroyo A, Valero J, Marrón T, Vidal C, Hontecillas B, Bernal J (1998) Pericardial fluid postmortem: comparative study of natural and violent deaths. Am J Forensic Med Pathol 19:266-268

18. Madea B, Kreuser C, Banaschak S (2001) Postmortem biochemical examination of synovial fluid - a preliminary study. Forensic Sci Int 118:29-35

19. Gagajewski A, Murakami MM, Kloss J, Edstrom M, Hillyer M, Peterson GF, Amatuzio J, Apple FS (2004) Measurement of chemical analytes in vitreous humor: stability and precision studies. J Forensic Sci 49:371-374

20. Arroyo A, Rosel P, Marrón T (2005) Cerebrospinal fluid: postmortem biochemical study. J Clin Forensic Med 12:153-156

21. Thierauf A, Musshoff F, Madea B (2009) Postmortem biochemical investigations of vitreous humor. Forensic Sci Int 192:78-82

22. Naumann HN (1949) Diabetes and uremia diagnosed at autopsy by testing cerebrospinal fluid and urine. Arch Pathol (Chic) 47:70-77
23. Naumann HN (1959) Postmortem chemistry of the vitreous body in man. Arch Ophthalmol 62:356-363

24. Coe JI (1972) Use of chemical determinations on vitreous humor in forensic pathology. J Forensic Sci 17:541-546

25. Coe JI (1973) Some further thoughts and observations on postmortem chemistries. Forensic Sci Gazette 5:2-6

26. Madea B, Lachenmeier DW (2005) Postmortem diagnosis of hypertonic dehydration. Forensic Sci Int 155:1-6

27. Leahy MS, Farber ER (1967) Postmortem chemistry of human vitreous humor. J Forensic Sci 12:214-222

28. Palmiere C, Mangin P (2012) Postmortem chemistry update part I. Int J Legal Med 126:187-198

29. Mitchell R, Charlwood C, Thomas SD, Bellis M, Langlois NE (2013) An audit of the contribution to post-mortem examination diagnosis of individual analyte results obtained from biochemical analysis of the vitreous. Forensic Sci Med Pathol 9:515-520

30. Tumram NK, Bardale RV, Dongre AP (2011) Postmortem analysis of synovial fluid and vitreous humour for determination of death interval: a comparative study. Forensic Sci Int 204:186-190

31. Li DR, Quan L, Zhu BL, Ishikawa T, Michiue T, Zhao D, Yoshida C, Chen JH, Wang Q, Komatsu A, Azuma Y, Maeda H (2009) Evaluation of postmortem calcium and magnesium levels in the pericardial fluid with regard to the cause of death in medicolegal autopsy. Forensic Sci Int 11(Suppl 1):S276-S278

32. Zhu BL, Ishikawa T, Michiue T, Li DR, Zhao D, Oritani S, Kamikodai Y, Tsuda K, Okazaki S, Maeda H (2006) Postmortem cardiac troponin $\mathrm{T}$ levels in the blood and pericardial fluid. Part 1. Analysis with special regard to traumatic causes of death. Legal Med (Tokyo) 8:86-93

33. Zhu BL, Ishikawa T, Michiue T, Li DR, Zhao D, Kamikodai Y, Tsuda K, Okazaki S, Maeda H (2006) Postmortem cardiac troponin T levels in the blood and pericardial fluid. Part 2: analysis for application in the diagnosis of sudden cardiac death with regard to pathology. Legal Med (Tokyo) 8:94-101

34. Zhu BL, Ishikawa $\mathrm{T}$, Michiue $\mathrm{T}$, Li DR, Zhao D, Bessho $\mathrm{Y}$, Kamikodai Y, Tsuda K, Okazaki S, Maeda H (2007) Postmortem cardiac troponin I and creatine kinase MB levels in the blood and pericardial fluid as markers of myocardial damage in medicolegal autopsy. Legal Med (Tokyo) 9:241-250

35. Wang Q, Michiue T, Ishikawa T, Zhu BL, Maeda H (2001) Combined analyses of creatine kinase MB, cardiac troponin I and myoglobin in pericardial and cerebrospinal fluids to investigate myocardial and skeletal muscle injury in medicolegal autopsy cases. Legal Med (Tokyo) 13:226-232

36. Quan L, Ishikawa T, Hara J, Michiue T, Chen JH, Wang Q, Zhu BL, Maeda H (2011) Postmortem serotonin levels in cerebrospinal and pericardial fluids with regard to the cause of death in medicolegal autopsy. Legal Med (Tokyo) 13:75-78

37. Ishikawa T, Quan L, Michiue T, Kawamoto O, Wang Q, Chen JH, Zhu BL, Maeda H (2013) Postmortem catecholamine levels in pericardial and cerebrospinal fluids with regard to the cause of death in medicolegal autopsy. Forensic Sci Int 228:52-60

38. Luna A, Villanueva E, Castellano M, Jimenez J (1982) The determination of $\mathrm{CK}, \mathrm{LDH}$ and its isoenzymes in pericardial fluid and its application to the post-mortem diagnosis of myocardial infarction. Forensic Sci Int 19:85-91

39. Luna A, Carmona A, Villanueva E (1983) The postmortem determination of CK isozymes in the pericardial fluid in various causes of death. Forensic Sci Int 22:23-30

40. Balasooriya BA, St Hill CA, Williams AR (1984) The biochemical changes in pericardial fluid after death. An investigation of the relationship between the time since death and the rise or fall in electrolyte and enzyme concentrations and their possible usefulness in determining the time of death. Forensic Sci Int 26:93-102

41. Valenzuela A, Hougen HP, Villanueva E (1994) Lipoproteins and apolipoproteins in pericardial fluid: new postmortem markers for coronary atherosclerosis. Forensic Sci Int 66:81-88 